\title{
Issues around host immunity
}

\author{
Morris Goldner PhD, \\ Faculté de médecine, Département de biologie médicale, Pavillon Ferdinand-Vandry, \\ Université Laval, Cité universitaire, Québec, Québec
}

$\mathrm{T}$ hese introductory comments are based on presentations by the following speakers at the Biological Implications of Pathogenicity 1999 (BIOP '99) Symposium, Towards Reducing Disease Transmission, Toronto, Ontario, February 25, 1999: Michael Hayek (Dayton, Ohio), Kelly MacDonald (Toronto) and David Westaway (Toronto), and on references from speakers at the BIOP '97 Symposium, Hygiene and Pathogenesis, Toronto, Ontario, May 2, 1997, notably Pierre Plourde (Winnipeg), Michael Eggert (Edmonton) and Gregor Reid (London).

The control of infectious diseases has increasingly been relegated to a secondary position in health research, somewhere behind cancer and cardiovascular pathologies. While fully recognizing the seriousness of the latter, to the careful observer, many infectious threats from the 19th and early 20th centuries appear to be regrouping and gaining virility, and certain infectious threats are newly emerging, at a time when our arsenal of effective antimicrobials is dangerously depleted. An intensified ecosystem approach might still yield greater understanding of the adversary.

Pathogenic organisms vary in their effects on the host, due in large part to variation in the host's response. It, therefore, becomes imperative to understand the subtlety of the host's susceptibility and defense mechanisms, and to integrate these concepts into creative strategies for reducing infectious disease. It is probable that the role of hygiene will become vigorously reemphasized in the prevention and control of infectious disease.

There is no doubt that we need a better understanding of the rule of 'balance' in a microbiological sense. At the mucosal surface, for example, the host must maintain active control of organisms. The mucosal surface provides a stage for the appearance of normal and infecting flora, as well as a site of interaction between an infectious process and host immunity. If the biological balance is maintained, this alludes to a role for some decisive factor on the part of the mucosal organisms inherent to a specific region. But if there is an imbalance, the conceptual explanation attributes a certain deficiency to the host.

Balance vis-à-vis imbalance clearly reflects the rapport between an infectious process and the host's immunity. Reinforcing that balance by instilling specially selected organisms might reasonably defend against an aggressive pathogenic assault. Such innocuous organisms, as probiotics, could be instilled into those affected areas normally inhabited by indigenous co-organisms.
In a deleterious agent, the existence of components homologous or analogous to the host tissue itself again addresses the subtle character of the host's immune function, but sometimes it may be aimed towards a subversion of host immunity. Recently, the 'infectious prion', related antigenically to the constitution of neuronal glycoprotein, has been presented as a conformational disorder of protein in its threedimensional structure (misfolding). It is acknowledged that the different patterns of prion disease manifestation can be traced to the biochemical properties of prion proteins. This proposes that the flawed protein provides a template for this conformational transition.

Forms of innate host control clearly influence the processes regarding infectious disease. For example, antioxidant supplementation (vitamin E) may decrease the impact of a viral infection. So, during a decline in immunity, the supply of certain nutrients above recognized requirements could improve the host's control, leading in turn to an improved immune response, ie, $T$ cell response and cytokine production, and allowing for the design of specific nutritional interventions.

In relation to innate host control, it can be presumed that antigens in place at the moment of disease transmission submit to the pressure of the host, target the interface with the host that senses the environmental surroundings and by their presence contribute to the pathogen's survival. An imbalance in the micro-environment at the interface with the host could either serve to advance the process of infection or induce particular antigenic changes that will negate the potential for transmissibility. As a proposition, an advantage of antigenic modulation as a reversible process could be its capacity to encompass the antigenic mode averting disease transmission, thus enhancing the host's capacity to avoid disease.

This commentary was inspired by the insight gained from the speakers at the BIOP symposia held in 1997 and 1999 in conjunction with the School of Occupational and Public Health, Ryerson Polytechnic University, Toronto, Ontario. The reader should refer to the original abstracts from the symposia (BIOP ' 97 Hygiene and Pathogenesis, May 2, 1997 and BIOP '99 Towards Reducing Disease Transmission, February 25, 1999 [Can J Infect Dis 1999;10:304306]), and to the "Virulence Modulon Theory of Pathogenicity" (Acta Biotheoretica 1997;45:81-85). The helpful criticism by Raina Fyson (Ottawa) and Timothy Sly (Toronto) is gratefully acknowledged. 


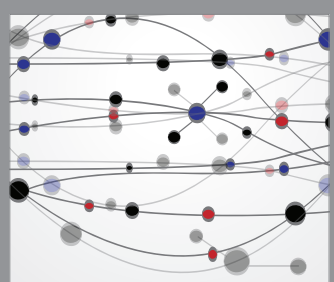

The Scientific World Journal
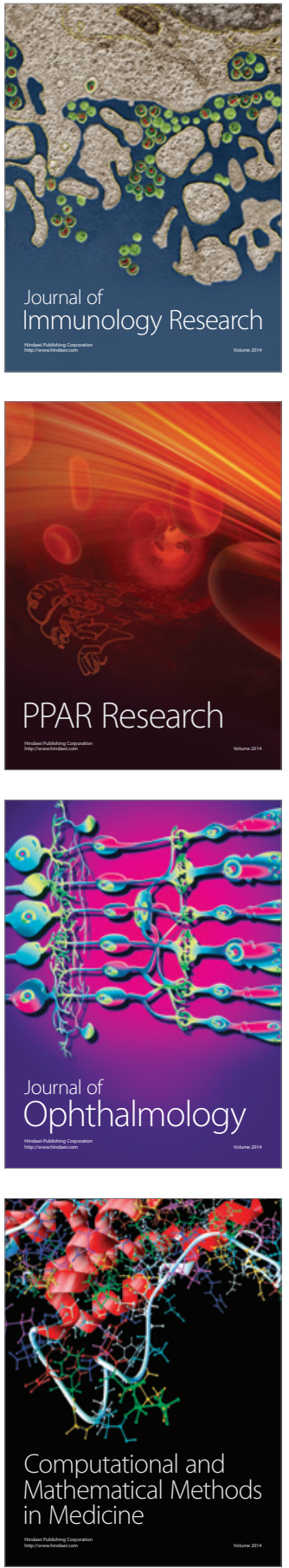

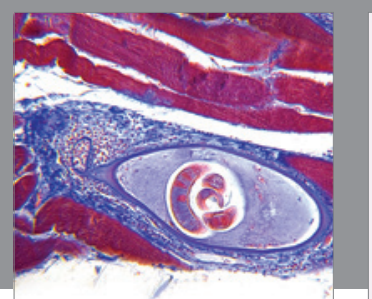

Gastroenterology Research and Practice

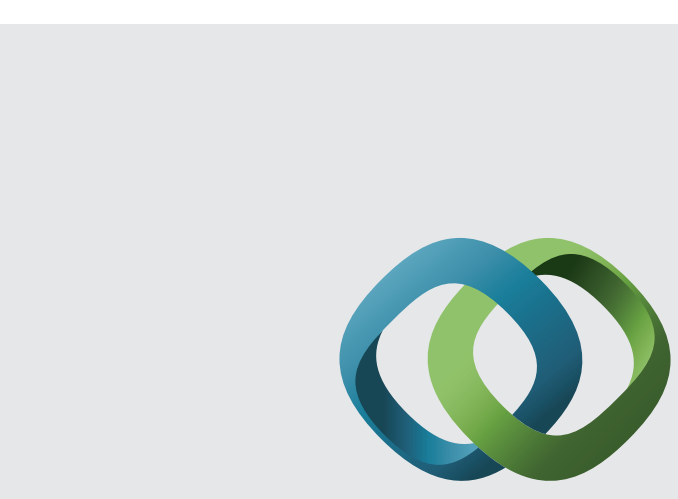

\section{Hindawi}

Submit your manuscripts at

http://www.hindawi.com
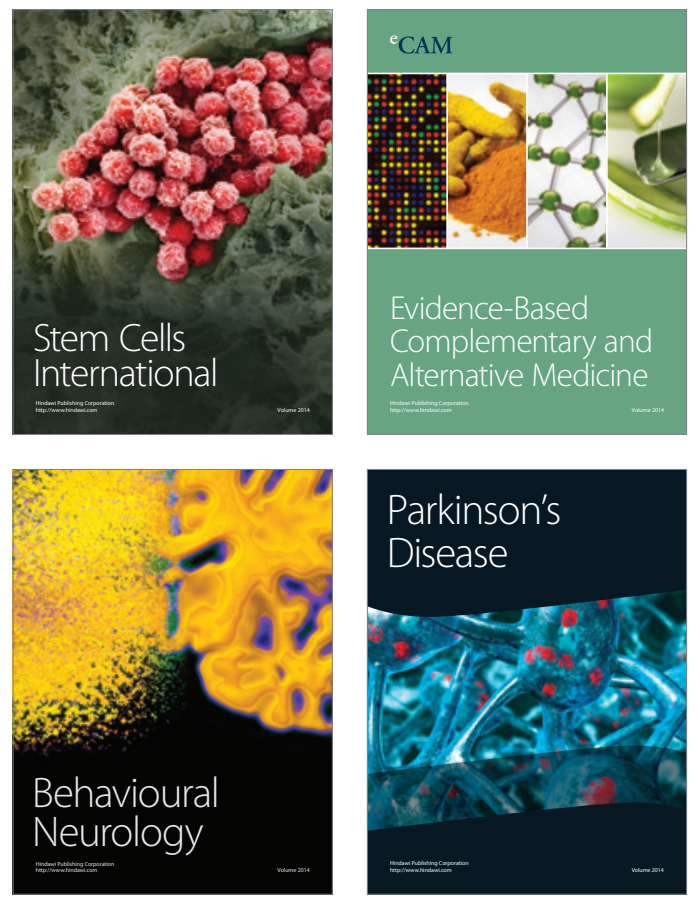
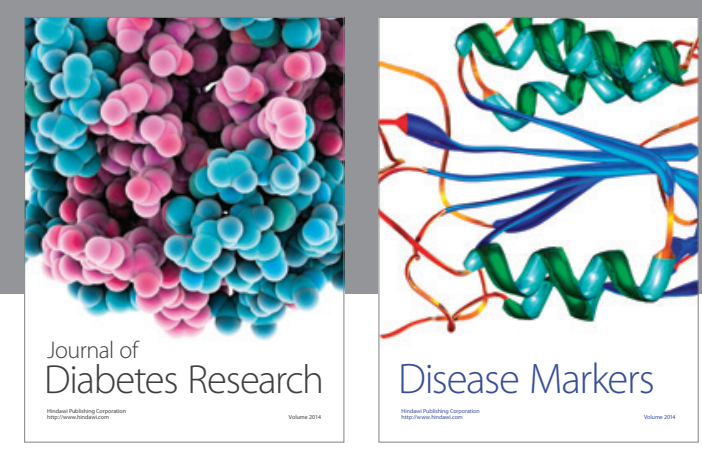

Disease Markers
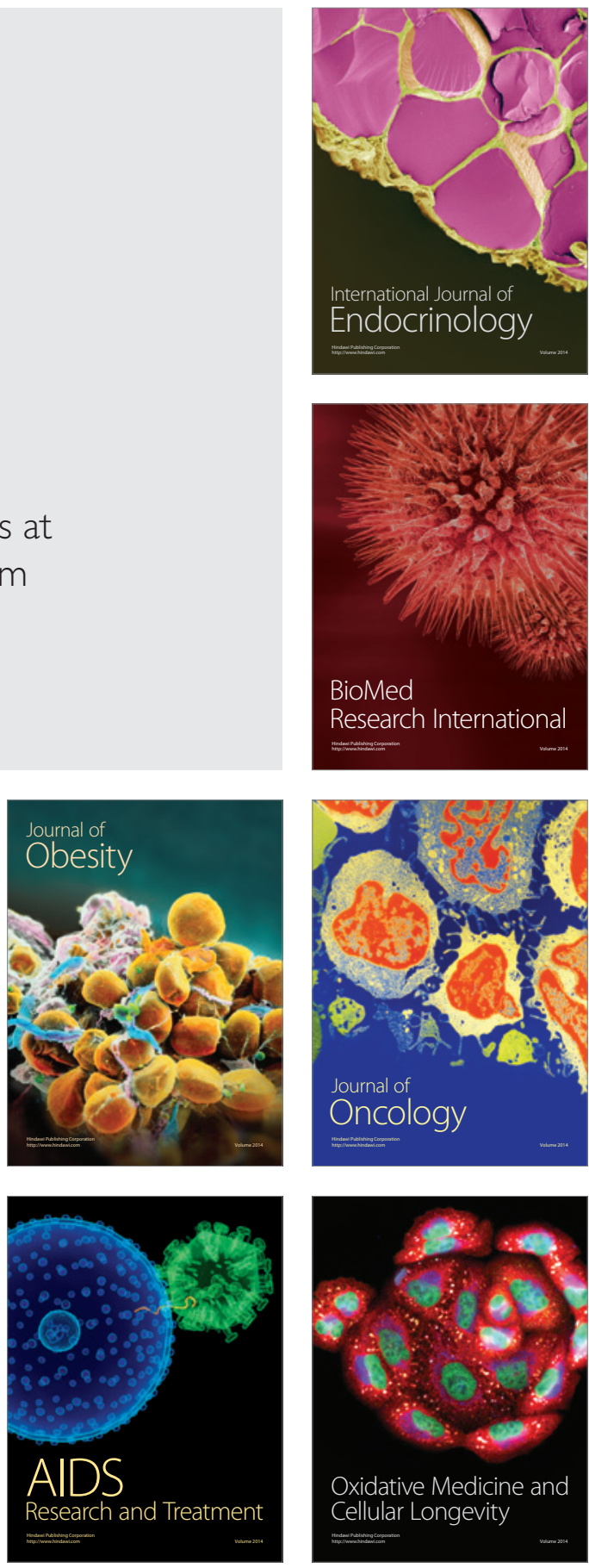